\title{
Innovation machine on the boil
}

Basic research and technological application are moving closer together.

\section{$\mathrm{O}$} Zeitschrift für Physik of an article with the cautious title "Possible high- $T_{\mathrm{c}}$ superconductivity in the Ba-La-Cu-O system". A few weeks later, the names of the two authors - Alexander Müller (pictured right) and Georg Bednorz (left) - and their discovery hit the front page of The New York Times. Researchers around the world were caught in an unprecedented frenzy, trying to replicate and surpass the findings of the initial breakthrough. The race for higher temperatures was on.

In early January 1987 Paul Chu found YBaCuO superconducting at about $90 \mathrm{~K}$. He immediately applied for a patent on the new compound, which marked a technological breakthrough because the critical temperature could now be attained with liquid nitrogen. The euphoria peaked during the annual meeting of the American Physical Society in March 1987, when almost 3,000 physicists squeezed into the New York Hilton hotel to hear the latest news - an event that became known as the "Woodstock of physics".

Things began to settle somewhat, at least in scientific circles, when in October 1987 Müller and Bednorz were awarded the Nobel Prize in Physics for the discovery of the first ceramic material to superconduct at the then high temperature of $30 \mathrm{~K}$. The award recognized the significance of their discovery, but it also meant that a stimulating race entered the next phase. It had become clear that years of more serious research still lay ahead before the new phenomenon would be fully understood. Surprisingly, less time would elapse before technologically reliable and commercially viable applications were to come. Views on how superconductivity is originated by electron-electron interactions, as opposed to electron-phonon interactions, are still unsettled. Theoretical understanding lags behind technological applications.

The discovery of high-temperature superconductivity (HTS) was unexpected in terms of its discoverers, the place of its discovery and the scientific ideas involved. It contradicted conventional wisdom and the expectations of peers and research administrators. Müller and Bednorz were outsiders; Müller an acknowledged specialist on perovskites, Bednorz a crystallographer. They benefited from the novice effect, but also enjoyed a degree of autonomy which, though temporary, allowed them to prepare for the unpredictable. Of IBM's three superconduc-
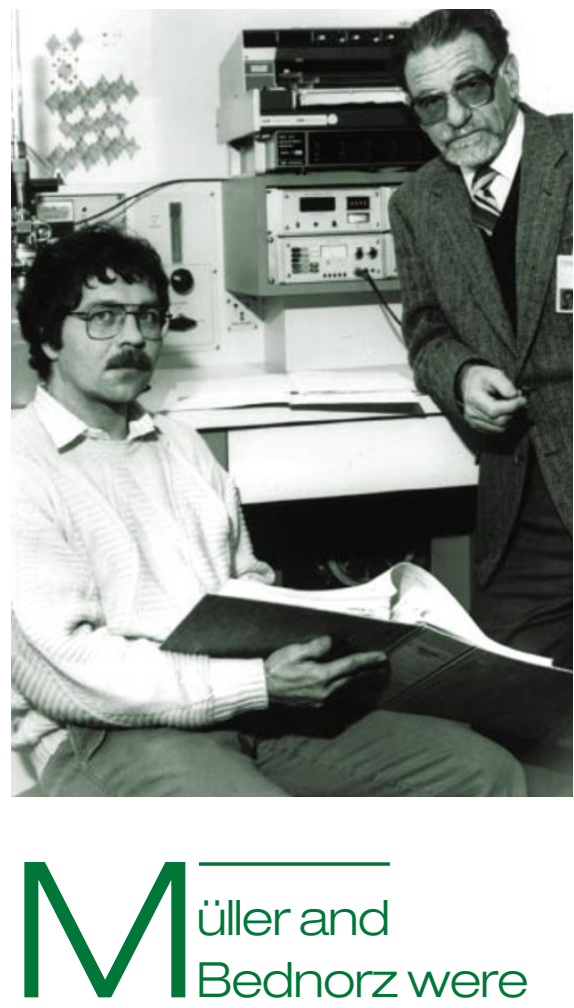

awarded a Nobel prize for the discovery of the first ceramic material to superconduct at $30 \mathrm{~K}$.

tivity laboratories, Rüschlikon near Zürich, where the two researchers were based, was by far the most modestly equipped. And the discovery contradicted long-held views. It not only overturned the established empirical rules of Bernd Matthias, one of the grand old experimentalists in superconductivity, but also unveiled previously unknown phenomena not accounted for by the BardeenCooper-Schrieffer theory.

But HTS also exemplifies the dramatic transformation that science is undergoing. The effects on the research system of the emergence of HTS can be likened to a building being tested by subjection to a transient load which reveals hidden strengths and weaknesses. The case of HTS shows how complex and fluid the situation has become. Researchers can no longer expect to find an environment unconditionally hospitable to their work. It takes an extraordinary amount of time and energy to set up the conditions under which research can run for a predictable period. Such efforts have become an integral feature of the work of researchers. Furthermore, nowhere is science policy firmly in place. It is continuously shaped and reshaped under the impact of new and the constraints of old requirements, such as shifting priorities, strategic thinking on how to combine knowledge-driven with missionoriented research, and how to accommodate objectives such as wealth creation.

HTS provides a fascinating glimpse into the present working of the scientific system as an 'innovation machine'. It reveals how basic research and technological applications are moving closer together and how the anticipation of technological potential affects the choice of what basic research is pursued. Basic research was once defined by psychologist Michael Posner as "that subset of what counts as good science which cannot be sold for immediate use". Yet HTS was 'sold' to the public — by the media, by politicians, by research-funding agencies and by the researchers themselves. All participants colluded in 'selling' the promise of technological advance as part of a widely shared belief. The case of HTS highlights the extent to which new criteria, extending beyond merely 'good science', have come into play and are effectively used in judging which areas of basic research should be funded.

The story of HTS also invites questions about the ideal conditions in which to foster individual creativity, and about how thin the line is between mere chance and programmed success. It probes the validity of Louis Pasteur's dictum that "chance favours only the prepared mind". It asks what preparedness means, not only on the individual level, but also on the institutional level. Even if we knew how to create conditions under which creativity can flourish, and how to favour the occurrence of what cannot be planned, the problem remains of how to turn highly individualistic bursts of scientific creativity into socially desired techno-scientific outcomes. For the most disturbing paradox is this: there has been a relative decline in the importance of the individual creative act, while its proliferation is encouraged. Individual scientific creativity has become a necessary, but no longer a sufficient, precondition in a long, branching sequence of possibilities. It has become contingent on what the innovation machinery of science will make of it as each new breakthrough is made.

Helga Nowotny is at the Swiss Federal Institute of Technology, ETH-Zentrum, CH-8092 Zürich, Switzerland. She and Ulrike Felt are the authors of After the Breakthrough (Cambridge Univ. Press, 1997). 